\title{
Association of nasopharyngeal microbiota profiles with bronchiolitis severity in infants hospitalised for bronchiolitis
}

\author{
Kohei Hasegawa (1) ${ }^{1}$, Jonathan M. Mansbach², Nadim J. Ajami ${ }^{3}$, \\ Janice A. Espinola1, David M. Henke ${ }^{4}$, Joseph F. Petrosino ${ }^{3}$, Pedro A. Piedra ${ }^{5}$, \\ Chad A. Shaw ${ }^{4}$, Ashley F. Sullivan ${ }^{1}$ and Carlos A. Camargo $\mathrm{Jr}^{1}$ on behalf of the \\ MARC-35 Investigators ${ }^{6}$
}

\begin{abstract}
Affiliations: ${ }^{1}$ Dept of Emergency Medicine, Massachusetts General Hospital, Harvard Medical School, Boston, MA, USA. ${ }^{2}$ Dept of Medicine, Boston Children's Hospital, Boston, MA, USA. ${ }^{3}$ Alkek Center for Metagenomics and Microbiome Research, Dept of Molecular Virology and Microbiology, Baylor College of Medicine, Houston, TX, USA. ${ }^{4}$ Dept of Molecular and Human Genetics, Baylor College of Medicine, Houston, TX, USA. ${ }^{5}$ Dept of Molecular Virology and Microbiology and Pediatrics, Baylor College of Medicine, Houston, TX, USA. ${ }^{6}$ A full list of the MARC-35 Investigators can be found in the acknowledgements section.
\end{abstract}

Correspondence: Kohei Hasegawa, Dept of Emergency Medicine, Massachusetts General Hospital, 125 Nashua Street, Suite 920, Boston, MA 02114, USA. E-mail: khasegawa1Apartners.org

ABSTRACT Little is known about the relationship between the specific airway microbiota composition and severity of bronchiolitis. We aimed to identify nasopharyngeal microbiota profiles and link these profiles to acute severity in infants hospitalised for bronchiolitis.

We conducted a multicentre prospective cohort study of 1005 infants (age <1 year) hospitalised for bronchiolitis over three winters, 2011-2014. By applying a 16S rRNA gene sequence and clustering approach to the nasopharyngeal aspirates collected within $24 \mathrm{~h}$ of hospitalisation, we determined nasopharyngeal microbiota profiles and their association with bronchiolitis severity. The primary outcome was intensive care use, i.e. admission to an intensive care unit or use of mechanical ventilation.

We identified four nasopharyngeal microbiota profiles: three profiles were dominated by one of Haemophilus, Moraxella or Streptococcus, while the fourth profile had the highest bacterial richness. The rate of intensive care use was highest in infants with a Haemophilus-dominant profile and lowest in those with a Moraxella-dominant profile (20.2\% versus $12.3 \%$; unadjusted OR $1.81,95 \%$ CI $1.07-3.11, \mathrm{p}=0.03)$. After adjusting for 11 patient-level confounders, the rate remained significantly higher in infants with Haemophilus-dominant profiles ( $\mathrm{OR} 1.98,95 \%$ CI 1.08-3.62, p=0.03). These findings were externally validated in a separate cohort of 307 children hospitalised for bronchiolitis.

@ERSpublications

Haemophilus-dominant airway microbiota associates with higher risk of intensive care in infants with bronchiolitis http://ow.ly/Bur6302vjRZ

This article has supplementary material available from erj.ersjournals.com

Received: Jan 202016 | Accepted after revision: July 182016 | First published online: Oct 062016

Support statement: This study was supported by the grants U01 AI-087881, R01 AI-114552, R01 AI-108588, and R21 HL-129909 from the National Institutes of Health (Bethesda, MD, USA) and a grant from the William F. Milton Fund (Boston, MA, USA). Funding information for this article has been deposited with the Open Funder Registry. The content of this manuscript is solely the responsibility of the authors and does not necessarily represent the official views of the National Institutes of Health.

Conflict of interest: Disclosures can be found alongside this article at erj.ersjournals.com

Copyright @ERS 2016 


\section{Introduction}

Bronchiolitis is a significant public health problem worldwide [1-3]. Indeed, bronchiolitis is the leading cause of hospitalisations for US infants, accounting for $18 \%$ of all infant hospitalisations [3]. The severity of bronchiolitis can range from a minor nuisance to fatal infection; however, the reasons for this severity difference are not fully explained by traditional risk factors, such as age, prematurity and viral aetiology $[4,5]$.

While common viruses and bacteria are identifiable using conventional techniques (e.g. cultures), these pathogens represent only a small fraction of the microbes living within any one individual $[6,7]$. The recent advances of genome sequencing have revealed the presence of highly functional bacterial communities inhabiting humans, i.e. the microbiota. Emerging evidence shows that airway microbiota can influence immune responses [8-13], suggesting a role for airway microbiota in the development and morbidity of acute respiratory infections (ARIs), including bronchiolitis [14]. However, the limited literature about the association of airway microbiota with ARI incidence during infancy is conflicting. Specifically, ARI incidence has been found to be lower [15] and higher [16] among infants with more abundant Moraxella genus in their nasopharynx. None of the aforementioned studies of airway microbiota have externally validated their findings. Moreover, to the best of our knowledge, no studies have investigated the airway microbiota and its relationship with the severity of illness in infants hospitalised for bronchiolitis; a vulnerable population with high morbidity.

In this context, we conducted a multicentre prospective cohort study of infants hospitalised for bronchiolitis to identify airway microbiota profiles and to link these profiles to acute severity (i.e. intensive care use and hospital length of stay). We also externally validated the findings in a separate multicentre study of children hospitalised for bronchiolitis.

\section{Materials and methods}

Study design, setting and participants

We conducted a multicentre prospective cohort study of infants (age $<1$ year) hospitalised for bronchiolitis (severe bronchiolitis). This study, called the 35th Multicenter Airway Research Collaboration (MARC-35) [17], was coordinated by the Emergency Medicine Network (EMNet, http://www.emnet-usa.org/), a collaboration of 235 participating hospitals.

Using a standardised protocol, site investigators at 17 sites across 14 US states (supplementary table E1) enrolled infants hospitalised with an attending physician diagnosis of bronchiolitis during three consecutive bronchiolitis seasons from November 1, to April 30, 2011-2014. Bronchiolitis was defined by the American Academy of Pediatrics guidelines as acute respiratory illness with some combination of rhinitis, cough, tachypnoea, wheezing, crackles and retractions [18]. We excluded infants with previous enrolment; those who were transferred to a participating hospital $>24 \mathrm{~h}$ after the original hospitalisation; those who were consented $>24 \mathrm{~h}$ after hospitalisation; or those with known heart-lung disease, immunodeficiency, immunosuppression or gestational age $<32$ weeks. All patients were treated at the discretion of the treating physician. The institutional review board at each of the participating hospitals approved the study. Written informed consent was obtained from the parent or guardian.

\section{Data collection}

Investigators conducted a structured interview that assessed patients' demographic characteristics, medical and family history, and details of the acute illness. Emergency department and hospital chart reviews provided further clinical data, such as vital signs, physical examination, medical management and disposition. All data were reviewed at the EMNet Coordinating Center and site investigators were queried about missing data and discrepancies identified by manual data checks.

Nasopharyngeal aspirates were collected by trained site investigators using the same standardised protocol utilised in a previous cohort study of children with bronchiolitis [4, 19]. All sites used the same collection equipment (Medline Industries, Mundelein, IL, USA) and collected the samples within $24 \mathrm{~h}$ of hospitalisation. The nasopharyngeal sample was added to transport medium, immediately placed on ice and then stored at $-80^{\circ} \mathrm{C}$. Frozen samples were shipped in batches on dry ice to Baylor College of Medicine, where they were tested for 1) 17 respiratory viruses (e.g. respiratory syncytial virus (RSV), rhinovirus) using real-time PCR assays [4, 19, 20], and 2) microbiota using $16 \mathrm{~S}$ rRNA gene sequencing.

\section{S rRNA gene sequencing and compositional analysis}

$16 \mathrm{~S}$ rRNA gene sequencing methods were adapted from the methods developed for the National Institutes of Health (NIH) Human Microbiome Project [6, 7]. The details of the methods are described in the supplemental methods. Briefly, bacterial genomic DNA was extracted using a MO BIO PowerSoil DNA Isolation Kit (Mo Bio Laboratories, Carlsbad, CA, USA). The $16 \mathrm{~S}$ rDNA V4 region was amplified by PCR and sequenced in the MiSeq platform (Illumina, SanDiego, CA, USA) using a $2 \times 250$ bp paired-end 
protocol that yields paired-end reads which overlap almost completely. The primers used for amplification contained adapters for MiSeq sequencing and single-end barcodes, allowing pooling and direct sequencing of PCR products [21, 22].

Sequencing read pairs were demultiplexed based on the unique molecular barcodes, and reads were merged using USEARCH v7.0.1090 [23]. Rarefaction curves of bacterial operational taxonomic units (OTUs) were constructed using sequence data for each sample to ensure coverage of the bacterial diversity present. Samples with suboptimal amounts of sequencing reads were re-sequenced to ensure that the majority of bacterial taxa were encompassed in our analyses.

16S rRNA gene sequences were clustered into OTUs at a similarity cut-off value of $97 \%$ using the UPARSE algorithm [24]. OTUs were determined by mapping the centroids to the SILVA database [25] containing only the $16 \mathrm{~S}$ V4 region to determine taxonomies. A custom script constructed a rarefied OTU table (rarefaction was performed at only one sequence depth) from the output files generated in the previous two steps for downstream analyses of alpha-diversity (i.e. Shannon index) and beta-diversity (i.e. weighted UniFrac) [26,27]. We utilised multiple quality control measures, including the use of non-template controls in the microbial DNA extraction, 16S rRNA gene amplification, and amplicon sequencing processes. The details of the quality control measures are described in the supplemental methods.

\section{Outcome measures}

The primary outcome was intensive care use, defined as admission to the intensive care unit and/or use of mechanical ventilation (continuous positive airway pressure and/or intubation during inpatient stay, regardless of location) at any time during the index hospitalisation $[19,28]$. The secondary outcome was hospital length of stay of $\geqslant 3$ days, defined using the median length of stay of 2 days and similar to the approach used in prior studies [4, 19].

\section{Statistical analyses}

For each nasopharyngeal sample, the relative abundance of each OTU was calculated. Analyses were conducted at the genus level; because each genus was dominated by one OTU (e.g. the detected Haemophilus genus consisted solely of one OTU), all OTUs assigned to the same genus were collapsed into a single group for reporting [16]. To identify nasopharyngeal microbiota profiles, we performed unbiased clustering by partitioning around medoids (PAM) [29] using weighted UniFrac distance. Each PAM cluster is defined by a point designated as the centre (the 'medoid') and minimises the distance between samples in a cluster. The number of clusters to choose for the data was determined using the average Silhouette score (supplementary figure E1) [30].

Additionally, given that the presence and/or abundance of an individual genus likely influences other genera in the microbial community, we displayed the microbiota association network on the basis of the approach of FAUST et al. [31]. The details of the method are described in the supplemental methods.

Next, across the identified nasopharyngeal microbiota profiles, we compared patient characteristics and hospital course using the Chi-squared test or Kruskal-Wallis test as appropriate. To examine the association of microbiota profiles with the severity outcomes (i.e. intensive care use and hospital length of stay of $\geqslant 3$ days), we constructed two logistic regression models for each outcome with the Moraxella-dominant profile as the reference [15]. First, we fitted an unadjusted model that included only microbiota profiles as the independent variable. Second, we constructed a two-level mixed-effects model to account for patient clustering at the hospital level. We also adjusted for 11 patient-level variables (i.e. age, sex, race/ethnicity, gestational age, history of breathing problems, nursery attendance, siblings at home, lifetime history of antibiotic use, history of corticosteroid use, use of antibiotics during the pre-hospitalisation visit and respiratory viruses detected by PCR). We chose these potential confounders on the basis of clinical plausibility and a priori knowledge $[4,5,19,28,32]$. We did not adjust for markers of severity (e.g. vital signs) because these were considered intermediate factors in the association of interest.

We performed sensitivity analyses to assess the robustness of our findings. First, we repeated the analysis stratifying by viral pathogen, similar to the approach used in prior studies [4, 33, 34]. Second, we modelled length of stay as a count variable by fitting a mixed-effects Poisson regression model. Analyses used R v3.2 with the lme4 package for the mixed-effects models and the phyloseq package [35]. All p-values were two-tailed, with $\mathrm{p}<0.05$ considered statistically significant.

\section{Validation of microbiota profiles and outcome models}

Next, to validate our findings in MARC-35, we analysed data from another 16-centre prospective cohort of children aged <2 years with severe bronchiolitis (MARC-30) [4, 19], in which nasopharyngeal samples were collected using the same methods as in MARC-35. The technical methods (i.e. microbial DNA 
extraction, 16S rRNA gene amplification and amplicon sequencing) used for MARC-30 were also identical to those for MARC-35. Using nasopharyngeal samples from 156 children with intensive care use and 156 children with no intensive care use and a length of stay $\leqslant 1$ day (i.e. nested case-control sample with a greater severity contrast), we tested for the nasopharyngeal microbiota, identified microbiota profiles, and determined the associations of these profiles with the severity outcomes.

\section{Results}

Population and sequence

We enrolled 1016 infants with severe bronchiolitis from 17 participating hospitals in MARC-35. We analysed nasopharyngeal samples from all of the enrolled infants by $16 \mathrm{~S}$ rRNA gene sequencing and obtained 17399260 high-quality merged sequences, of which 16685637 (95.9\%) were mapped to the database. Of 1016 infant samples, 1005 (98.9\%) had sufficient sequence depth (rarefaction cut-off: 2128 reads per sample) and were eligible for the current analysis. Among the analytic cohort, the median age at hospitalisation was 3 months (interquartile range (IQR), 2-6 months), $60.0 \%$ were male and $42.6 \%$ were non-Hispanic white. The sequencing identified 24 phyla and 379 genera. The nasopharyngeal microbiota was dominated by three genera: Streptococcus (30.9\%), Moraxella (29.8\%) and Haemophilus (20.2\%); followed by Prevotella (2.3\%) and Staphylococcus (2.2\%).

\section{Nasopharyngeal microbiota profiles and network}

PAM clustering of nasopharyngeal microbiota identified four distinct microbiota profiles: 1) Haemophilusdominant profile $(19.2 \%), 2)$ Moraxella-dominant profile $(21.9 \%), 3)$ Streptococcus-dominant profile $(28.2 \%)$ and 4$)$ a mixed profile (30.7\%) (figure 1$)$.

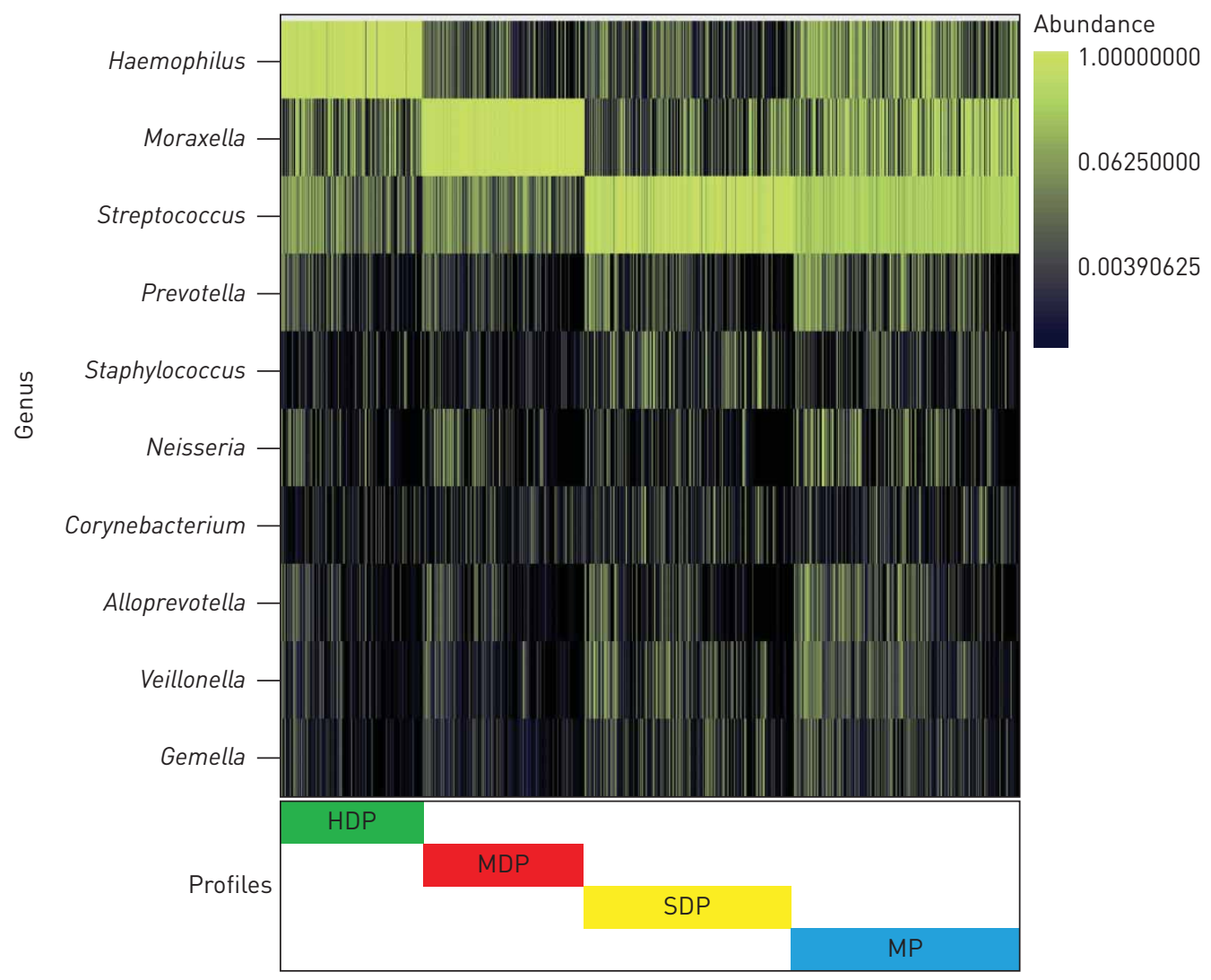

FIGURE 1 Clustering and composition in nasopharyngeal microbiota of 1005 infants hospitalised for bronchiolitis, MARC-35. All nasopharyngeal microbiota profiles of infants were clustered using the partitioning around medoids clustering method with weighted UniFrac distance. Coloured bars indicate four microbiota profiles: Haemophilus-dominant profile (HDP, green), Moraxella-dominant profile (MDP, red), Streptococcus-dominant profile (SDP, yellow), and mixed profile (MP, blue). The optimal number of clusters was identified by the Silhouette index. To obtain further information about the bacterial composition of samples within microbiota profiles, the 10 most abundant genera present are displayed in the adjacent heatmap. The taxonomy depicted is on the genus level because each genus was dominated by one operational taxonomic unit. 
The first three profiles were dominated by one of Haemophilus, Moraxella or Streptococcus genera. By contrast, the mixed profile had the highest bacterial richness $(\mathrm{p}<0.001)$ and alpha-diversity index (Shannon index, $\mathrm{p}<0.001$ ) with highest relative abundance of Prevotella, Alloprevotella and Veillonella (supplementary table E2). Sub-clustering of the mixed profile identified three sub-clusters: a sub-cluster co-dominated by Streptococcus and Moraxella, a sub-cluster co-dominated by Streptococcus and Haemophilus, and a sub-cluster with a relatively high abundance of Streptococcus, Prevotella and Alloprevotella (supplementary table E3).

The presence or abundance of individual bacteria likely affects those of others due to ecological interactions. To examine the community structure of the microbiota, we created a microbiota association network (supplementary figure E2). Haemophilus, Moraxella and Streptococcus genera were negatively associated with each other, which is consistent with the observed Haemophilus-dominant, Moraxella-dominant and Streptococcus-dominant profiles. The Streptococcus genus was positively associated with Veillonella, which was also positively associated with Prevotella and Alloprevotella. This community structure was consistent with the mixed microbiota profile.

\section{Microbiota profiles and patient characteristics}

Patient characteristics differed across the four microbiota profiles (table 1). For example, infants with the Haemophilus-dominant profile were older, less likely to be non-Hispanic white and more likely to have used antibiotics before the index hospitalisation compared to infants with other profiles (all $\mathrm{p}<0.05)$. At presentation to the hospital, infants with the Haemophilus-dominant profile also had a higher weight and were more likely to receive antibiotics during a pre-hospitalisation visit (e.g. to the emergency department before enrolment) and have sole rhinovirus infection (all $\mathrm{p}<0.001$ ), compared to infants with other profiles. By contrast, there was no significant difference in the vital signs across the profiles.

\section{Microbiota profiles and bronchiolitis severity}

Overall, 161 (16.0\%) infants hospitalised for bronchiolitis required intensive care use. The rate of intensive care use was highest in infants with a Haemophilus-dominant profile and lowest in those with a Moraxella-dominant profile (20.2\% versus $12.3 \%$; unadjusted OR 1.81, 95\% CI 1.07-3.11, p=0.03; table 2). In the multivariable model adjusting for 11 patient characteristics (e.g., demographics, use of antibiotics during the pre-hospitalisation visit, virus) and clustering at the hospital level, the rate remained significantly higher in infants with a Haemophilus-dominant profile (OR for comparison with Moraxella-dominant profile 1.98, 95\% CI 1.08-3.62, p=0.03; table 2 and supplementary table E4). There was also a positive linear relationship between relative abundance of Haemophilus and rate of intensive care use after adjusting for the 11 patient characteristics $(p=0.03$; figure $2 a)$. By contrast, the rate of intensive care use in those with a Streptococcus-dominant or mixed profile was not significantly different from that of infants with the reference Moraxella-dominant profile. Stratified analyses across virology strata are shown in supplementary table E5. Although there was limited statistical power in most viral strata, infants with RSV infection and a Haemophilus-dominant profile had higher odds of intensive care use. Additionally, in another sensitivity analysis $(n=978)$ that excluded all infants with no viral pathogen detected, the results did not change materially, i.e. the rate of intensive care use remained significantly higher in infants with a Haemophilus-dominant profile (OR for comparison with Moraxella-dominant profile 2.15, 95\% CI 1.16-3.99, $\mathrm{p}=0.01$ ).

Similar to results of the primary outcome, infants with a Haemophilus-dominant profile had a higher rate of length of stay of $\geqslant 3$ days than did those with the Moraxella-dominant profile $(51.3 \%$ versus $33.2 \%$; unadjusted OR 2.12, 95\% CI 1.43-3.17, p<0.001; tables 1 and 2). In the multivariable model, the rate remained significantly higher in infants with a Haemophilus-dominant profile (OR 2.47, 95\% CI 1.60 3.83, $\mathrm{p}<0.001$; table 2 and supplementary table E4). There was also a positive linear relationship between relative abundance of Haemophilus and rate of length-of-stay $\geqslant 3$ days $(\mathrm{p}<0.001$; figure $2 \mathrm{~B})$. Likewise, in the sensitivity analysis modelling length of stay as a count variable (i.e. not as binary variable), the significant association between microbiota profiles and the outcome persisted (supplementary table E6).

\section{Validation of profiles and association with severity}

In 312 nasopharyngeal samples selected from children in the MARC-30 study, 307 (98.4\%) had sufficient sequencing depth (rarefaction cut-off: 1064 reads per sample) and were used for validation. The use of the PAM clustering method of microbiota, with the use of the average Silhouette score, revealed four microbiota profiles (supplementary figure E3) similar to those in MARC-35, i.e. three profiles were dominated by one of Haemophilus, Moraxella or Streptococcus genera, while the fourth profile (i.e. mixed profile) had the highest bacterial richness and alpha-diversity levels (supplementary table E7). In addition, the sub-clustering of the mixed profile identified a sub-cluster with a relatively high abundance of Streptococcus, Prevotella and Alloprevotella (supplementary table E8). Likewise, the significant associations 
TABLE 1 Characteristics and clinical presentation of infants hospitalised for bronchiolitis by nasopharyngeal microbiota profile in the MARC-35 cohort

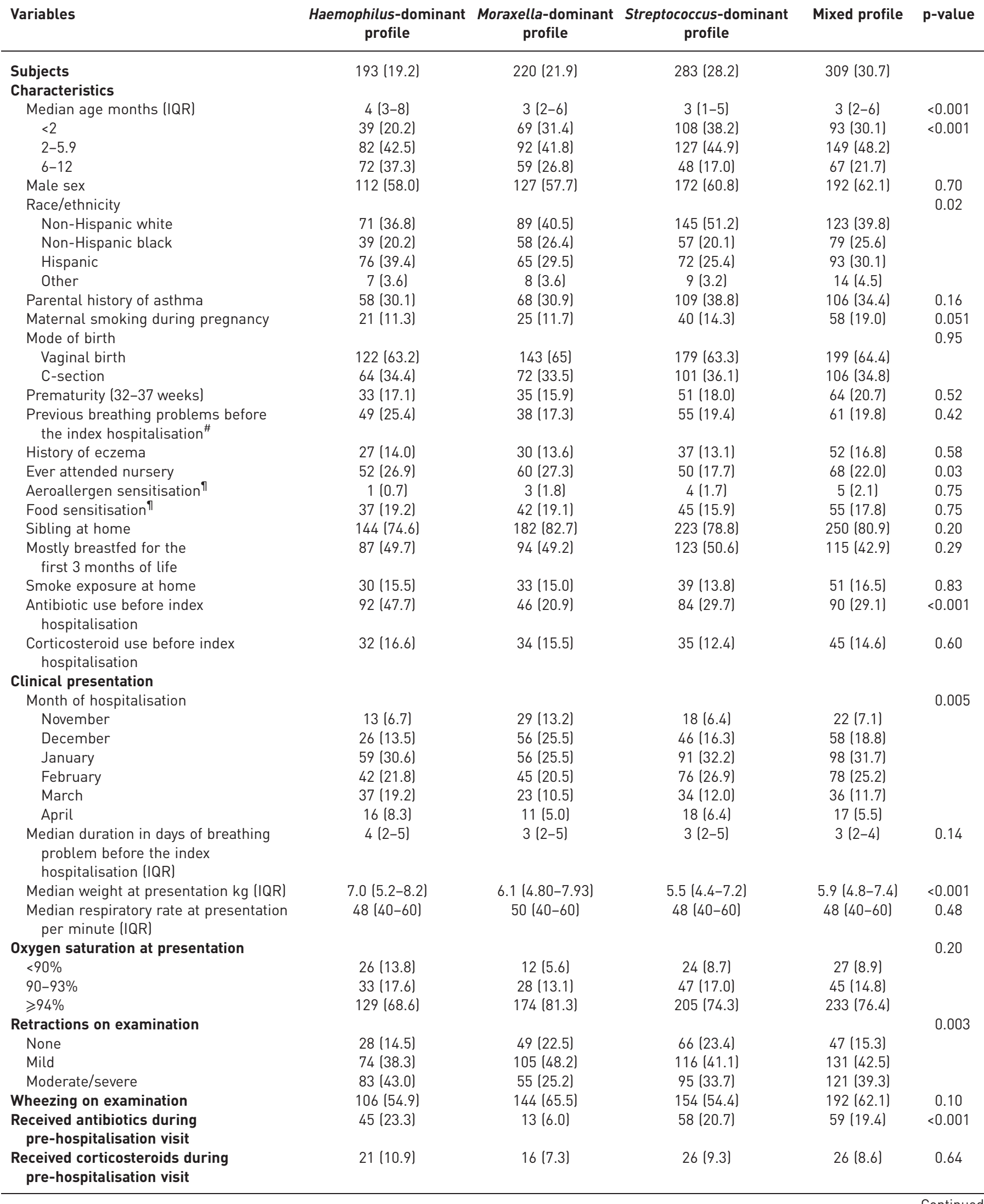


TABLE 1 Continued

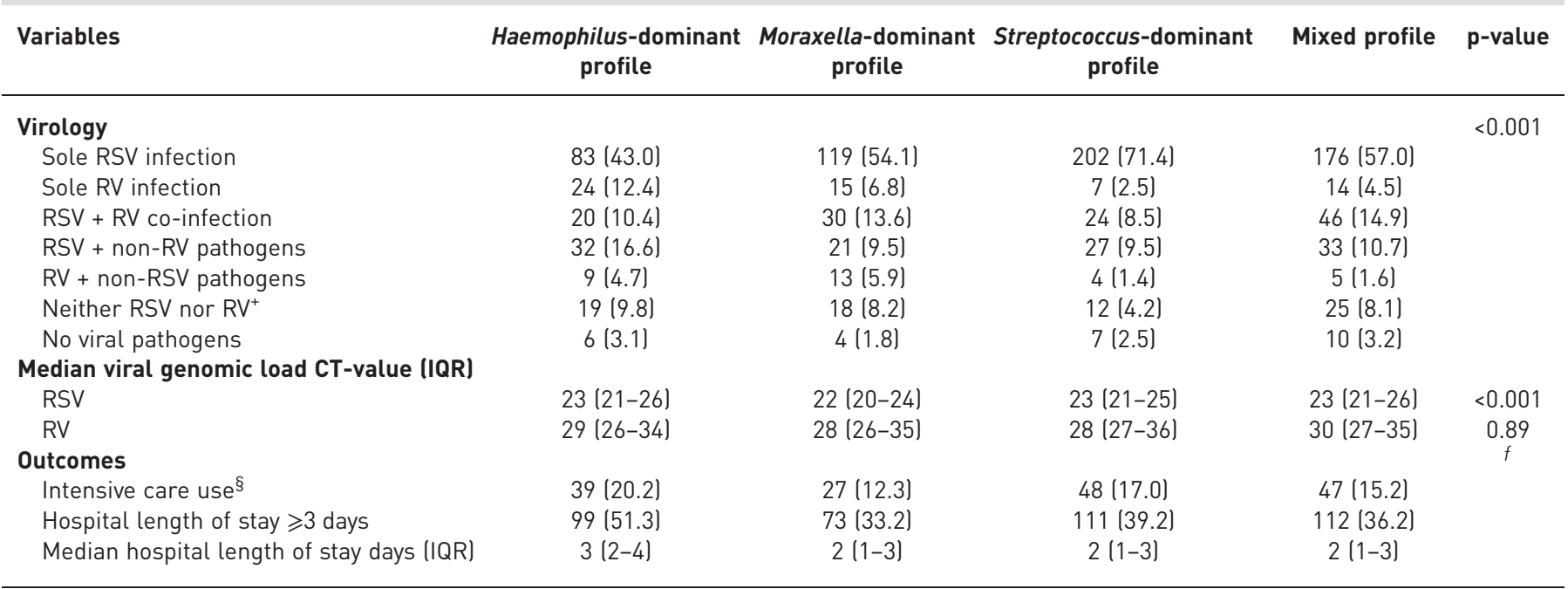

Data are presented as $\mathrm{n}(\%)$ of infants unless otherwise indicated. Percentages may not equal 100, because of missingness. Patient characteristics and hospital course were compared using the Chi-squared test or Kruskal-Wallis test across the identified nasopharyngeal microbiota profiles. IQR: interquartile range; RSV: respiratory syncytial virus; RV: rhinovirus; CT: cycle threshold. " : Defined as an infant having cough that wakes him/her at night and/or causes emesis, or when the child has wheezing or shortness of breath without cough. ": Defined by having one or more positive values for allergen-specific IgE. ${ }^{+}$: Consisted of adenovirus ( $\left.n=6\right)$, coronavirus ( $\left.n=15\right)$, human bocavirus ( $n=9$ ), human metapneumovirus ( $n=38)$, influenza virus $(n=11)$, and parainfluenza virus $(n=13)$. Note that 18 patients had co-infection. $\$$ : Defined as admission to the intensive care unit and/or use of mechanical ventilation (continuous positive airway pressure and/or intubation during inpatient stay, regardless of location) at any time during the index hospitalisation. ${ }^{f}$ : Unadjusted $p$-values are presented in table 2.

between the Haemophilus-dominant profile and the higher rate of severity outcomes were also observed, with larger effect sizes, in this nested case-control sample with a greater severity contrast, for example, the adjusted odds ratio for intensive care use of Haemophilus-dominant profile in comparison with Moraxella-dominant profile was 5.34 (95\% CI 1.96-14.5, p=0.001; supplementary table E9).

\section{Discussion}

In this prospective multicentre cohort of 1005 infants with severe bronchiolitis, we identified four distinct microbiota profiles in their nasopharynx. Infants with a Haemophilus-dominant profile had significantly higher rates of intensive care use and prolonged hospital length of stay than those with a Moraxella-dominant profile. In contrast, the rate of outcomes in those with a Streptococcus-dominant or mixed profile was not significantly different. These findings were externally validated in a separate multicentre study of severe bronchiolitis. To our knowledge, this is the first study to have examined the association of airway microbiota with disease severity in infants with severe bronchiolitis. Our data corroborate and build on previous reports linking bacteria composition in the airway to ARI outcomes $[15,16,36-39]$, findings of both research and clinical importance.

Previous reports on ARI in young children have reported inconsistent relationships between airway bacteria and ARI incidence and severity. For example, KLOEPFER et al. [36] applied quantitative PCR to nasal samples $(\mathrm{n}=380)$ and found no associations between $H$. influenzae detection alongside rhinovirus and ARI severity in school-age children. Similarly, CARLSSON et al. [37], using a culture-dependent technique in the COPSAC ${ }_{2000}$ cohort $(\mathrm{n}=283)$, found no association between $H$. influenzae detection in the hypopharynx and duration of wheezing episodes in children aged $<3$ years. By contrast, TEO et al., using 16S rRNA gene sequencing in the Childhood Asthma Study cohort $(\mathrm{n}=234)$ in Western Australia, reported six nasopharyngeal microbiota profiles (Haemophilus, Moraxella, Streptococcus, Corynebacterium, Alloiococcus and Staphylococcus) in infants with a high risk of atopy, and that a Haemophilus-dominant nasopharyngeal microbiota was associated with higher incidence of ARI and higher severity [16]. In addition to the apparent inconsistency in the Haemophilus-ARI link, we also observed a lower abundance of Corynebacterium, Dolosigranulum and Staphylococcus genera in our population (i.e. young infants hospitalised with bronchiolitis) compared to that in previous studies of healthy children [15] and children with mild upper respiratory infection [40,41]. Potential reasons for these discrepancies across studies include differences in patient populations, clinical settings (e.g. community versus inpatient setting), sampling (e.g. anterior nasal swab versus nasopharyngeal aspirate) and laboratory techniques (e.g. culture, PCR) for bacteria identification. By contrast, the validity of our findings is buttressed by the use of $16 \mathrm{~S}$ 
TABLE 2 Unadjusted and multivariable associations of nasopharyngeal microbiota profiles with bronchiolitis outcomes, MARC-35 cohort

Outcome by microbiota profile Unadjusted model

Adjusted model ${ }^{\#}$

\begin{tabular}{|c|c|c|c|c|}
\hline & & \\
\hline & OR $(95 \% \mathrm{CI})$ & p-value & OR $(95 \% \mathrm{CI})$ & p-value \\
\hline \multicolumn{5}{|l|}{ Intensive care use ${ }^{\Uparrow}$} \\
\hline Haemophilus-dominant profile & $1.81(1.07-3.11)$ & 0.03 & $1.98(1.08-3.62)$ & 0.03 \\
\hline Moraxella-dominant profile & Reference & & Reference & \\
\hline Streptococcus-dominant profile & $1.46(0.88-2.45)$ & 0.14 & $1.32(0.74-2.34)$ & 0.34 \\
\hline Mixed profile & $1.28(0.78-2.16)$ & 0.34 & $1.19(0.68-2.09)$ & 0.54 \\
\hline \multicolumn{5}{|l|}{ Hospital length of stay $\geqslant 3$ days } \\
\hline Haemophilus-dominant profile & $2.12(1.43-3.17)$ & $<0.001$ & $2.47(1.60-3.83)$ & $<0.001$ \\
\hline Moraxella-dominant profile & Reference & & Reference & \\
\hline Streptococcus-dominant profile & $1.30(0.90-1.88)$ & 0.16 & $1.06(0.71-1.57)$ & 0.78 \\
\hline Mixed profile & $1.14(0.80-1.65)$ & 0.47 & $1.01(0.68-1.48)$ & 0.97 \\
\hline
\end{tabular}

Bold results are statistically significant. Full adjusted models are included in supplementary table E3.

\#: Mixed-effects logistic regression model adjusting for 11 patient-level variables lage, sex, race/ethnicity, gestational age, history of breathing problems, nursey attendance, siblings at home, lifetime history of antibiotic use, history of corticosteroid use, use of antibiotics during the pre-hospitalisation visit and respiratory viruses detected by PCR) and sites as random effect. ": Defined as admission to the intensive care unit and/or use of mechanical ventilation (continuous positive airway pressure and/or intubation during inpatient stay, regardless of location) at any time during the index hospitalisation.

rRNA gene sequencing of the nasopharyngeal microbiota, a sample size that is many times larger than any other prior study on this topic, and validation in an additional multicentre cohort of severe bronchiolitis.

The observed microbiota-severity association challenges the conventional virus-centric view of bronchiolitis. However, the nature of this microbial association warrants clarification. It is possible that there is a causal relationship, i.e. the Haemophilus-dominant microbiota in the infant's airway alters immune responses to increase the severity of bronchiolitis. Indeed, FøLSGAARD et al. reported that $H$. influenzae colonisation of the airways in asymptomatic neonates is associated with an upregulated T-helper (Th)1/Th2/Th17-type inflammatory response of the upper airway mucosa [11]. The presence of
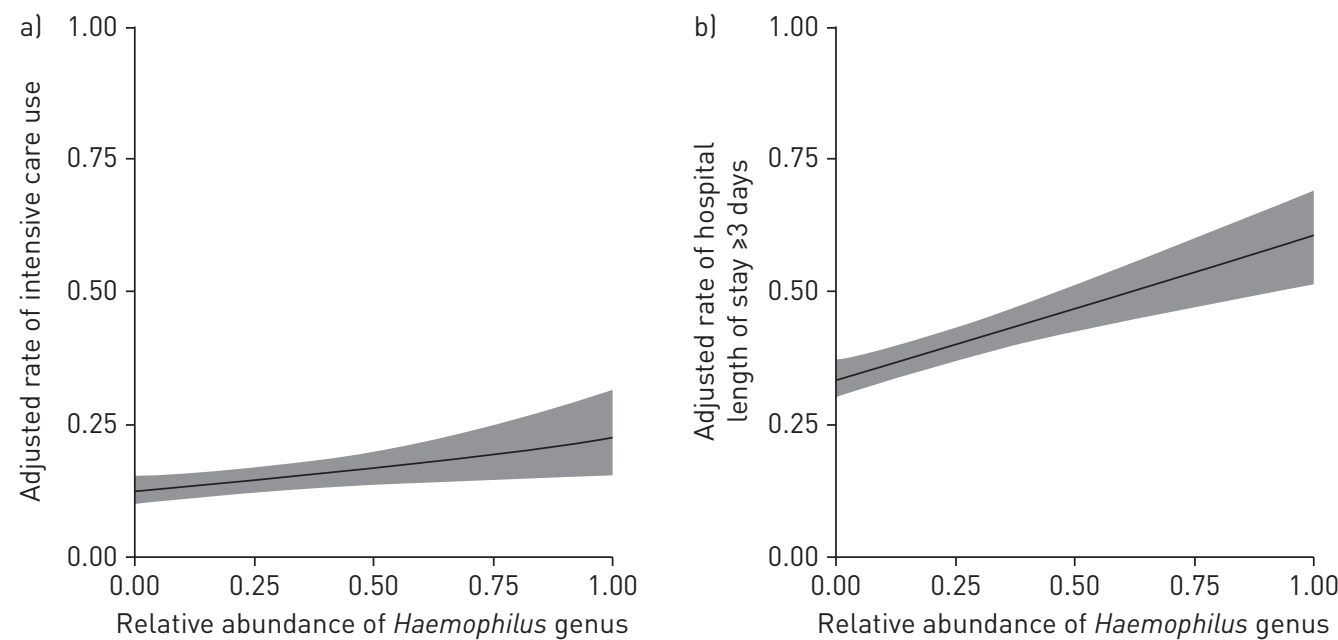

FIGURE 2 Multivariable association of relative abundance of Haemophilus genus with the rate of severity outcomes in infants hospitalised for bronchiolitis, MARC-35. Two-level mixed-effects models were constructed to account for patient clustering at the hospital level. The models adjusted for 11 patient-level variables (i.e. age, sex, race/ethnicity, gestational age, history of breathing problems, nursery attendance, siblings at home, lifetime history of antibiotic use, history of corticosteroid use, use of antibiotics during the pre-hospitalisation visit and respiratory viruses detected by PCR). a) There was a positive linear association between relative abundance of Haemophilus genus and the rate of intensive care use ladjusted OR 1.07 [per 0.1 increase in the relative abundance of Haemophilus], $95 \% \mathrm{Cl} 1.01-1.13, p=0.03$ ). b) There was a positive linear association between relative abundance of Haemophilus genus and the rate of a hospital length of stay of $\geqslant 3$ days ladjusted OR 1.11 [per 0.1 increase in the relative abundance of Haemophilus], $95 \% \mathrm{Cl} 1.06-1.17, \mathrm{p}<0.001$ ). The grey shaded areas represent the $95 \%$ confidence intervals. 
both Th2 and Th17 mediators suggests that colonisation with $H$. influenzae could counteract the Th1 response needed to eradicate viral pathogens of bronchiolitis. Similarly, studies of primary bronchial epithelial cells demonstrated that exposure to $H$. influenzae upregulates intercellular adhesion molecule-1 expression and enhances chemokine release induced by subsequent infection by RSV [42] and rhinovirus $[42,43]$. Alternatively, a Haemophilus-dominant microbiota could simply be a marker of an infant who is prone to develop more-severe bronchiolitis. Additionally, reverse causation, i.e. more-severe illness that results in a rapid overgrowth of Haemophilus in the airway, is also possible [44]. These possibilities are not mutually exclusive. Notwithstanding the complexity, the identification and validation of a Haemophilus-dominant profile as the primary culprit in the association between airway microbiota and bronchiolitis severity is an important finding. Our data should facilitate further investigations (e.g. animal models, metagenomics, proteomics, metabolomics) to disentangle the complex web of the airway microbiome, viral pathogens, host immune responses, and bronchiolitis pathogenesis [14].

\section{Potential limitations}

The study has several potential limitations. First, bronchiolitis is a disease of the lower airways, and our study was based on samples obtained from the infant's nasopharynx. However, lower airway sampling in infants is technically and ethically challenging. Nevertheless, prior studies have shown strong correlations between upper and lower airway microbiology [45] and virology [46] in children. Therefore, we believe that the nasopharyngeal microbiota in the infant is likely indicative of that in the lower respiratory tract. Second, with the use of $16 \mathrm{~S}$ rRNA gene sequencing, we did not analyse the microbiota at the species level; therefore, we were limited in the confirmation of their phenotypic features. Third, the study design precluded us from evaluating the relationship between succession of the airway microbial ecosystem and respiratory health in children. To address this important question, the study population is currently being followed longitudinally up to age 6 years with airway specimen sampling at multiple time points. Fourth, as with any observational studies, the associations of microbiota profiles with bronchiolitis severity do not necessarily prove causality and could be confounded by unmeasured factors such as virus genotypes and institutional variation in resource use. One could surmise that our inferences are biased by co-circulating viruses within hospitals or regions. However, MARC-35 enrolled patients at 17 hospitals across 14 US states across three consecutive bronchiolitis seasons (2011-2014). Their findings were further validated in a separate 16-centre cohort of severe bronchiolitis during 2007-2010. Furthermore, the observed association between the microbiota and bronchiolitis severity remained significant after accounting for patient-level clustering within hospitals using a mixed-effects model. Fifth, we did not have information from a 'control' group (e.g. infants hospitalised for non-respiratory events). However, the study objective was not to assess the role of microbiota on the development of bronchiolitis (yes/no) but to investigate its relationship with disease severity. Finally, although the study cohorts consisted of a racially, ethnically and geographically diverse US sample of severe bronchiolitis, our inferences might not be generalisable to those in the ambulatory setting. Nevertheless, our data remain highly relevant for 130000 children hospitalised yearly in the USA; a vulnerable population with high morbidity [3].

\section{Conclusions}

In this prospective multicentre cohort of 1005 infants hospitalised for bronchiolitis, we identified four distinct microbiota profiles in their nasopharynx. Infants with a Haemophilus-dominant profile had greater bronchiolitis severity, i.e. significantly higher rates of intensive care use and prolonged length of stay, than those with a Moraxella-dominant profile. These findings were validated in a separate multicentre study of severe bronchiolitis. The findings should serve as an important starting point for further mechanistic and interventional investigations of the interplay between the airway microbiome, viral pathogens, host immune responses and bronchiolitis pathogenesis.

\section{Acknowledgments}

We thank Pamela Luna at Rice University (Houston, TX, USA) for her assistance with statistical analysis.

The following individuals were MARC-35 site investigators and are collaborators on this paper. Anne K. Beasley, Phoenix Children's Hospital, Phoenix, AZ, USA; Juan C. Celedon, Children's Hospital of Pittsburgh, Pittsburgh, PA, USA; Ari R. Cohen, Massachusetts General Hospital, Boston, MA, USA; Michelle B. Dunn, Children's Hospital of Philadelphia, Philadelphia, PA, USA; Michael Gomez, Children's Hospital at St. Francis, Tulsa, OK, USA; Nancy Inhofe, Children's Hospital at St. Francis, Tulsa, OK, USA; Sujit Iyer, Dell Children's Medical Center of Central Texas, Austin, TX, USA; Federico R. Laham, Arnold Palmer Hospital for Children, Orlando, FL, USA; Charles G. Macias, Texas Children's Hospital, Houston, TX, USA; Thida Ong, Seattle Children's Hospital, Seattle, WA, US; Brian M. Pate, The Children's Mercy Hospital \& Clinics, Kansas City, MO, USA; Wayne G. Schreffler, Massachusetts General Hospital, Boston, MA, USA; Michelle D. Stevenson, Kosair Children's Hospital, Louisville, KY, USA; Richard T. Strait, Cincinnati Children's Hospital and Medical Center, Cincinnati, OH, USA; Stephen J. Teach, Children's National Medical Center, Washington, DC, USA; Henry T. Puls, The Children's Mercy Hospital \& Clinics, Kansas City, MO, US; Amy D. Thompson, Alfred I. duPont Hospital for Children, Wilmington, DE, USA; Vincent J. Wang, Children's Hospital of Los Angeles, Los Angeles, CA, USA; and Ilana Waynik, MD, Connecticut Children's Medical Center, Hartford, CT, USA. 


\section{References}

1 Nair H, Nokes DJ, Gessner BD, et al. Global burden of acute lower respiratory infections due to respiratory syncytial virus in young children: a systematic review and meta-analysis. Lancet 2010; 375: 1545-1555.

2 Hasegawa K, Tsugawa Y, Brown DF, et al. Temporal trends in emergency department visits for bronchiolitis in the United States, 2006-2010. Pediatr Infect Dis J 2014; 33: 11-18.

3 Hasegawa K, Tsugawa Y, Brown DF, et al. Trends in bronchiolitis hospitalizations in the United States, 2000-2009. Pediatrics 2013; 132: 28-36.

4 Mansbach JM, Piedra PA, Teach SJ, et al. Prospective multicenter study of viral etiology and hospital length of stay in children with severe bronchiolitis. Arch Pediatr Adolesc Med 2012; 166: 700-706.

5 Hasegawa K, Mansbach JM, Camargo CA Jr. Infectious pathogens and bronchiolitis outcomes. Exp Rev Anti Infect Ther 2014; 12: 817-828.

6 Human Microbiome Project Consortium. A framework for human microbiome research. Nature 2012; 486: 215-221.

7 Human Microbiome Project Consortium. Structure, function and diversity of the healthy human microbiome. Nature 2012; 486: 207-214

8 Herbst T, Sichelstiel A, Schar C, et al. Dysregulation of allergic airway inflammation in the absence of microbial colonization. Am J Respir Crit Care Med 2011; 184: 198-205.

9 Olszak T, An D, Zeissig S, et al. Microbial exposure during early life has persistent effects on natural killer T cell function. Science 2012; 336: 489-493.

10 Hollams EM, Hales BJ, Bachert C, et al. Th2-associated immunity to bacteria in teenagers and susceptibility to asthma. Eur Respir J 2010; 36: 509-516.

11 Folsgaard NV, Schjorring S, Chawes BL, et al. Pathogenic bacteria colonizing the airways in asymptomatic neonates stimulates topical inflammatory mediator release. Am J Respir Crit Care Med 2013; 187: 589-595.

12 Lynch SV. Viruses and microbiome alterations. Ann Am Thorac Soc 2014; 11: Suppl. 1, S57-S60.

13 Huang YJ, Nariya S, Harris JM, et al. The airway microbiome in patients with severe asthma: associations with disease features and severity. J Allergy Clin Immunol 2015; 136: 874-884.

14 Hasegawa K, Camargo CA, Jr. Airway microbiota and acute respiratory infection in children. Exp Rev Clin Immunol 2015; 11: 789-792.

15 Biesbroek G, Tsivtsivadze E, Sanders EA, et al. Early respiratory microbiota composition determines bacterial succession patterns and respiratory health in children. Am J Respir Crit Care Med 2014; 190: 1283-1292.

16 Teo SM, Mok D, Pham K, et al. The infant nasopharyngeal microbiome impacts severity of lower respiratory infection and risk of asthma development. Cell Host Microbe 2015; 17: 704-715.

17 Mansbach JM, Hasegawa K, Henke DM, et al. Respiratory syncytial virus and rhinovirus severe bronchiolitis are associated with distinct nasopharyngeal microbiota. J Allergy Clin Immunol 2016; 137: 1909-1913.

18 Ralston SL, Lieberthal AS, Meissner HC, et al. Clinical practice guideline: the diagnosis, management, and prevention of bronchiolitis. Pediatrics 2014; 134: e1474-e1502.

19 Hasegawa K, Jartti T, Mansbach JM, et al. Respiratory syncytial virus genomic load and disease severity among children hospitalized with bronchiolitis: multicenter cohort studies in the US and Finland. J Infect Dis 2015; 211: 1550-1559.

20 Beckham JD, Cadena A, Lin J, et al. Respiratory viral infections in patients with chronic, obstructive pulmonary disease. J Infect 2005; 50: 322-330.

21 Caporaso JG, Kuczynski J, Stombaugh J, et al. QIIME allows analysis of high-throughput community sequencing data. Nat Methods 2010; 7: 335-336.

22 Caporaso JG, Lauber CL, Walters WA, et al. Ultra-high-throughput microbial community analysis on the Illumina HiSeq and MiSeq platforms. ISME J 2012; 6: 1621-1624.

23 Edgar RC. Search and clustering orders of magnitude faster than Blast. Bioinformatics 2010; 26: 2460-2461.

24 Edgar RC. Uparse: Highly accurate OTU sequences from microbial amplicon reads. Nat Methods 2013; 10: 996-998.

25 Quast C, Pruesse E, Yilmaz P, et al. The SILVA ribosomal RNA gene database project: improved data processing and web-based tools. Nucleic Acids Res 2013; 41: D590-D596.

26 Lozupone C, Lladser ME, Knights D, et al. UniFrac: an effective distance metric for microbial community comparison. ISME J 2011; 5: 169-172.

27 Lozupone C, Knight R. UniFrac: a new phylogenetic method for comparing microbial communities. Appl Environ Microbiol 2005; 71: 8228-8235.

28 Jartti T, Hasegawa K, Mansbach JM, et al. Rhinovirus-induced bronchiolitis: lack of association between virus genomic load and short-term outcomes. J Allergy Clin Immunol 2015; 136: 509-512.

29 Wu GD, Chen J, Hoffmann C, et al. Linking long-term dietary patterns with gut microbial enterotypes. Science 2011; 334: 105-108.

30 Rousseeuw PJ. Silhouettes: a graphical aid to the interpretation and validation of cluster analysis. J Computational Appl Math 1987; 20: 53-65.

31 Faust K, Sathirapongsasuti JF, Izard J, et al. Microbial co-occurrence relationships in the human microbiome. PLoS Comput Biol 2012; 8: e1002606.

32 Mansbach JM, Piedra PA, Stevenson MD, et al. Prospective multicenter study of children with bronchiolitis requiring mechanical ventilation. Pediatrics 2012; 130: e492-e500.

33 Hasegawa K, Mansbach JM, Teach SJ, et al. Multicenter study of viral etiology and relapse in hospitalized children with bronchiolitis. Pediatr Infect Dis J 2014; 33: 809-813.

34 Hasegawa K, Stevenson MD, Mansbach JM, et al. Association between hyponatremia and higher bronchiolitis severity among children in the ICU with bronchiolitis. Hosp Pediatr 2015; 5: 385-389.

35 McMurdie PJ, Holmes S. phyloseq: an R package for reproducible interactive analysis and graphics of microbiome census data. PLoS One 2013; 8: e61217.

36 Kloepfer KM, Lee WM, Pappas TE, et al. Detection of pathogenic bacteria during rhinovirus infection is associated with increased respiratory symptoms and asthma exacerbations. J Allergy Clin Immunol 2014; 133: 1301-1307.

37 Carlsson CJ, Vissing NH, Sevelsted A, et al. Duration of wheezy episodes in early childhood is independent of the microbial trigger. J Allergy Clin Immunol 2015; 136: 1208-1214. 
38 Vissing NH, Chawes BL, Bisgaard H. Increased risk of pneumonia and bronchiolitis after bacterial colonization of the airways as neonates. Am J Respir Crit Care Med 2013; 188: 1246-1252.

39 von Linstow ML, Schonning K, Hoegh AM, et al. Neonatal airway colonization is associated with troublesome lung symptoms in infants. Am J Respir Crit Care Med 2013; 188: 1041-1042.

40 Pettigrew MM, Laufer AS, Gent JF, et al. Upper respiratory tract microbial communities, acute otitis media pathogens, and antibiotic use in healthy and sick children. Appl Environ Microbiol 2012; 78: 6262-6270.

41 Laufer AS, Metlay JP, Gent JF, et al. Microbial communities of the upper respiratory tract and otitis media in children. mBio 2011; 2: e00245-e00210.

42 Gulraiz F, Bellinghausen C, Bruggeman CA, et al. Haemophilus influenzae increases the susceptibility and inflammatory response of airway epithelial cells to viral infections. FASEB J 2015; 29: 849-858.

43 Sajjan US, Jia Y, Newcomb DC, et al. H. influenzae potentiates airway epithelial cell responses to rhinovirus by increasing ICAM-1 and TLR3 expression. FASEB J 2006; 20: 2121-2123.

44 McGillivary G, Mason KM, Jurcisek JA, et al. Respiratory syncytial virus-induced dysregulation of expression of a mucosal beta-defensin augments colonization of the upper airway by non-typeable Haemophilus influenzae. Cell Microbiol 2009; 11: 1399-1408.

45 Charlson ES, Bittinger K, Haas AR, et al. Topographical continuity of bacterial populations in the healthy human respiratory tract. Am J Respir Crit Care Med 2011; 184: 957-963.

46 Mosser AG, Vrtis R, Burchell L, et al. Quantitative and qualitative analysis of rhinovirus infection in bronchial tissues. Am J Respir Crit Care Med 2005; 171: 645-651. 